\title{
SIMPLE LOCALLY FINITE LIE ALGEBRAS OF DIAGONAL TYPE
}

\author{
A.A. BARANOV \\ Dedicated with admiration to Helmut Strade on the occasion of his 70th birthday
}

\begin{abstract}
We discuss various characterizations of simple locally finite Lie algebras of diagonal type over an algebraically closed field of characteristic zero.
\end{abstract}

\section{INTRODUCTION}

Throughout the paper, the ground field $\mathbb{F}$ is assumed to be algebraically closed of characteristic zero. Recall that an algebra $A$ is called locally finite if every finitely generated subalgebra of $A$ is finite dimensional. All locally finite algebras considered in the paper are assumed to be infinite dimensional. Let $L$ be a locally finite Lie algebra. If $L$ is of countable dimension then it has a chain of finite dimensional subalgebras

$$
L_{1} \subseteq L_{2} \subseteq \cdots \subseteq L_{n} \subseteq \cdots
$$

such that $L=\bigcup_{n=1}^{\infty} L_{n}$. We can also say that $L$ is the direct $\operatorname{limit} \lim _{n} L_{n}$ of the sequence of the embeddings (1.1). This also works in the general case: every locally finite Lie algebra $L$ can be represented as the direct $\operatorname{limit} \underset{\lim }{\longrightarrow} L_{\alpha}$ where $\left(L_{\alpha}\right)_{\alpha \in \Gamma}$ is a local system of finite dimensional subalgebras of $L$ (see Definition $2 . \overrightarrow{1}$ ). The standard way to deal with locally finite Lie algebras is to find nice local systems. For example, it is helpful when all $L_{\alpha}$ are semisimple. We say that $L$ is locally simple (semisimple, solvable, etc) if $L$ has a local system $\left(L_{\alpha}\right)_{\alpha \in \Gamma}$ such that all $L_{\alpha}$ are simple (semisimple, solvable, etc).

In this paper we focus on simple locally finite Lie algebras. Although the full classification of simple locally finite Lie algebras seems to be impossible to obtain, there are two classes of these algebras which have especially nice properties and can be characterized in many different ways. Those are finitary simple Lie algebras and diagonal simple locally finite Lie algebras. Recall that an infinite dimensional Lie algebra is called finitary if it consists of finite-rank linear transformations of a vector space. It is easy to see that finitary Lie algebras are locally finite. Finitary simple Lie algebras were completely classified in [8] (zero characteristic) and [17] (positive characteristic). In particular, there are just three finitary simple Lie algebras over $\mathbb{F}$ of infinite countable dimension: $s l_{\infty}(\mathbb{F}), s o_{\infty}(\mathbb{F})$ and $s p_{\infty}(\mathbb{F})$. It follows from the classification that finitary simple Lie algebras are locally semisimple and diagonal.

Locally finite Lie algebras of diagonal type were introduced in [6] and are defined as the limits of "diagonal" embeddings of finite dimensional Lie algebras (see Definition 2.3 for details). They have many interesting characterizations, which are discussed in this paper. To define diagonal embeddings it is best to consider classical simple Lie algebras first: $s l_{n}(\mathbb{F}), s o_{n}(\mathbb{F})$ and $s p_{n}(\mathbb{F})$. Suppose all algebras $L_{n}$ in (1.1) are classical simple. Denote by $V_{n}, V_{n}^{*}$ and $T_{n}$ the natural $L_{n}$-module, its dual and the trivial 1-dimensional $L_{n}$-module, respectively. We say that the embedding $L_{n} \rightarrow L_{n+1}$ is natural if the restriction $V_{n+1} \downarrow L_{n} \cong V_{n} \oplus T_{n} \oplus \cdots \oplus T_{n}$, i.e. the $L_{n}$-module $V_{n+1}$ contains exactly one nontrivial composition factor and this factor is isomorphic to $V_{n}$. We say that the embedding $L_{n} \rightarrow L_{n+1}$ is diagonal if the $L_{n}$-module $V_{n+1}$ 
is diagonal, i.e.

$$
V_{n+1} \downarrow L_{n} \cong \underbrace{V_{n} \oplus \cdots \oplus V_{n}}_{l_{n}} \oplus \underbrace{V_{n}^{*} \oplus \cdots \oplus V_{n}^{*}}_{r_{n}} \oplus \underbrace{T_{n} \oplus \cdots \oplus T_{n}}_{z_{n}}
$$

for some non-negative integers $l_{n}, r_{n}$ and $z_{n}$. In particular, an embedding $\varphi_{n}: \operatorname{sl}\left(V_{n}\right) \rightarrow$ $s l\left(V_{n+1}\right)$ is diagonal if and only if there exist bases of $V_{n}$ and $V_{n+1}$ such that $\varphi$ has the following matrix form:

$$
\varphi_{n}: X \mapsto \operatorname{diag}(\underbrace{X, \ldots, X}_{l_{n}}, \underbrace{-X^{t}, \ldots,-X^{t}}_{r_{n}}, \underbrace{0, \ldots, 0}_{z_{n}})
$$

where $X^{t}$ is the transpose of the matrix $X$. Diagonal embeddings can also be defined for nonsemisimple $L_{n}$ (see the beginning of Section 2). A simple locally finite Lie algebra is called diagonal if it has a local system of subalgebras such that the corresponding embeddings are diagonal.

Example 1.1. (1) The Lie algebra $s l_{\infty}$ is defined as the limit of the sequence of natural embeddings $\varphi_{n}: s l_{n} \rightarrow s l_{n+1}$ where $\varphi_{n}(X)=\operatorname{diag}(X, 0)$ for all $n \times n$ matrices $X$ with zero trace. Similarly one can define the algebras $s o_{\infty}$ and $s p_{\infty}$.

(2) The Lie algebra $s l_{2}$ is defined as the limit of the sequence of diagonal embeddings $\varphi_{n}: s l_{2^{n}} \rightarrow s l_{2^{n+1}}$ where $\varphi_{n}(X)=\operatorname{diag}(X, X)$.

(3) The previous example can be generalized as follows. Let $\mathcal{N}=\left(l_{1}, l_{2}, l_{3}, \ldots\right)$ be a sequence of positive integers and let $q_{n}=l_{1} l_{2} \ldots l_{n}$. Define $s l_{\mathcal{N}}$ as the limit of the sequence of diagonal matrix embedding $\varphi_{n}: s l_{q_{n}} \rightarrow s l_{q_{n+1}}$ where $\varphi_{n}(X)=\operatorname{diag}(X, \ldots, X)\left(l_{n+1}\right.$ copies $)$.

(4) The Lie algebra $s l_{(1,-1)^{\infty}}$ is defined as the limit of the sequence of embeddings $\varphi_{n}$ : $s l_{2^{n}} \rightarrow s l_{2^{n+1}}$ where $\varphi_{n}(X)=\operatorname{diag}\left(X,-X^{t}\right)$.

(5) The Lie algebra $s l_{a d}$ is defined as the limit of the sequence of embeddings

$$
s l_{2} \rightarrow s l\left(s l_{2}\right) \cong s l_{3} \rightarrow s l\left(s l_{3}\right) \cong s l_{8} \rightarrow \ldots
$$

where all embeddings are induced by the adjoint map $x \mapsto \operatorname{ad} x$.

(6) All previous examples can be generalized as follows. Let the sequences $\varphi=\left(V_{1}, V_{2}, V_{3}, \ldots\right)$ and $\mathcal{L}=\left(L_{1}, L_{2}, L_{3}, \ldots\right)$ be constructed inductively as follows: $V_{1}$ is any finite dimensional vector space, $L_{n}$ is the Lie algebra $s l\left(V_{n}\right)$ and $V_{n+1}$ is any non-trivial $L_{n}$-module for $n=1,2,3, \ldots$ Then we have a sequence of embeddings $L_{1} \rightarrow L_{2} \rightarrow L_{3} \rightarrow \ldots$ and the corresponding direct limit $s l_{\varphi}=\lim _{n} L_{n}$ is a simple locally finite Lie algebra.

Note that all Lie algebras in Example 1.1 are locally finite and simple (since they are the unions of simple subalgebras). Moreover, the algebras (1)-(4) are diagonal and the algebra $s l_{a d}$ (and $s l_{\varphi}$ in the case of infinitely many non-diagonal embeddings) is non-diagonal (see Corollary 2.11). Since almost all irreducible representations of the classical Lie algebras are non-diagonal, we have a lot of choice for $V_{n}$ in Example 1.1(6) to obtain a non-diagonal Lie algebra. This suggests that almost all simple locally finite Lie algebras are non-diagonal. Even in the diagonal case, as it was shown by Bahturin and Strade in their pioneering paper [4], there are uncountably many pairwise non-isomorphic algebras of type $s l_{\mathcal{N}}$ (as defined in Example 1.1(3)). Diagonal locally simple Lie algebras of countable dimension were completely classified in [20] (see also [9] for the analogue in positive characteristic). They are parametrized by two real numbers and two Steinitz (or supernatural) numbers (infinite products of the form $p_{1}^{\alpha_{1}} p_{2}^{\alpha_{2}} p_{3}^{\alpha_{3}} \ldots$ where $p_{1}, p_{2}, p_{3}, \ldots$ is the sequence of all primes and $\alpha_{i}=0,1,2, \ldots, \infty$ for all $i)$. As a very special case of the classification one gets the following. 
Proposition 1.2. [20] Let $\mathcal{N}=\left(l_{1}, l_{2}, l_{3}, \ldots\right)$ and $\mathcal{N}^{\prime}=\left(l_{1}^{\prime}, l_{2}^{\prime}, l_{3}^{\prime}, \ldots\right)$ be two sequences of positive integers. Then the Lie algebras $s l_{\mathcal{N}}$ and $s l_{\mathcal{N}^{\prime}}$ are isomorphic if and only if the infinite products $l_{1} l_{2} l_{3} \cdots=l_{1}^{\prime} l_{2}^{\prime} l_{3}^{\prime} \ldots$ as Steinitz numbers.

Note that the paper [20] doesn't exhaust the classification problem even in the diagonal case because of the following result by Bahturin and Strade.

Theorem 1.3. [5] There exists a simple diagonal locally finite Lie algebra that is not locally semisimple.

Another example of a simple diagonal locally finite Lie algebra that is not locally semisimple was constructed later by Zalesski [37]. These examples show that we need to deal with nonsemisimple local systems of subalgebras if we wish to study simple locally finite Lie algebras in full generality.

A full classification of simple diagonal locally finite Lie algebras was obtained in [2]. We need some notation to state the result. Let $A$ be an associative algebra over $\mathbb{F}$ and let $A^{(-)}$be the associated Lie algebra (vector space $A$ with the Lie product $[a, b]=a b-b a$ ). We define by $[A, A]$ the derived subalgebra of $A^{(-)}$. If $A$ has an involution $*$ of the first kind (i.e. an $\mathbb{F}$-linear antiautomorphism of order 2), then $K=\operatorname{skew}(A)=\left\{a \in A \mid a^{*}=-a\right\}$ is a Lie algebra over $\mathbb{F}$ with respect to the Lie product. It is not difficult to check that if $A$ is a simple (resp. involution simple) locally finite associative algebra then the Lie algebra $[A, A]$ (resp. $[K, K]$ ) is simple, locally finite and diagonal (actually, the simplicity of these algebras follows from the very general result by Herstein [32]). It turns out that every diagonal simple locally finite Lie algebra is obtained in this way.

Theorem 1.4. [2] Every diagonal simple locally finite Lie algebra is isomorphic to $[A, A]$ or $[K, K]$ for some (involution) simple locally finite associative algebra.

The proof of Theorem 1.4 is based on some technical results obtained in several other papers (mainly $[6,7,18,19]$ ). There is an alternative approach which is based on Zelmanov's famous classification of simple Lie algebras with finite gradings [38]. This approach was recently used by Hennig [31] to obtain an analogue of Theorem 1.4 in positive characteristic and provide a new proof in characteristic zero.

We need some notation to state the main theorem.

If $S$ is a finite dimensional Lie algebra and $V$ is an $S$-module then $\operatorname{Irr}(S)$ denotes the set of all simple finite dimensional $S$-modules up to isomorphism and $\operatorname{Irr}(V) \subseteq \operatorname{Irr}(S)$ denotes the set of all composition factors of $V$ up to isomorphism (disregarding the multiplicities).

Let $L$ be a locally finite Lie algebra. An $L$-module $V$ is called integrable if for any $v \in V$ and any finite dimensional subalgebra $S$ of $L$ one has $\operatorname{dim} U(S) v<\infty$, or equivalently, $V$ is the direct limit of its finite dimensional $S$-submodules. An $L$-module $V$ is said to be of finite type if it is integrable and the set $\operatorname{Irr}(V \downarrow S)$ is finite for every finite-dimensional subalgebra $S$ of $L$. Let $\left(L_{\alpha}\right)_{\alpha \in \Gamma}$ be a local system of $L$ and let $\Phi_{\alpha}$ be a non-empty finite subset of $\operatorname{Irr}\left(L_{\alpha}\right)$, $\alpha \in \Gamma$. The set $\Phi=\left(\Phi_{\alpha}\right)_{\alpha \in \Gamma}$ is called an inductive system of representations for $L$ if

$$
\cup_{V \in \Phi_{\beta}} \operatorname{Irr}\left(V \downarrow L_{\alpha}\right)=\Phi_{\alpha} \text { for all } \alpha \leq \beta .
$$

Let $U(L)$ be the universal enveloping algebra of a Lie algebra $L$ and let $A(L)$ be its augmentation ideal (the ideal of codimension 1 generated by $L$ ). We say that an ideal $Q$ of $U(L)$ is proper if $Q \neq U(L), A(L)$. An element $x$ of a Lie algebra $L$ is called Jordan if $(\operatorname{ad} x)^{3}=0$ (i.e. $x$ is ad-nilpotent of degree 3 ). A subspace $B$ of Lie algebra $L$ is called an inner ideal if $[B,[B, L]] \subseteq B$ 
A $\mathbb{Z}$-grading of an algebra $A$ is a decomposition into a sum of subspaces $A=\sum_{i \in \mathbb{Z}} A_{i}$ such that $A_{i} A_{j} \subseteq A_{i+j}$. Such a grading is finite if the set $\left\{i \in \mathbb{Z} \mid A_{i} \neq 0\right\}$ is finite and the grading is nontrivial if $\sum_{i \neq 0} A_{i} \neq 0$.

We are now ready to state the main theorem.

Theorem 1.5. Let $L$ be an infinite dimensional simple locally finite Lie algebra over $\mathbb{F}$. Then the following are equivalent.

(1) $L$ is diagonal.

(2) [2] $L=[A, A]$ or $[K, K]$ for some (involution) simple locally finite associative algebra $A(K=\operatorname{skew}(A)$ if $A$ has an involution).

(3) [7] $L$ is isomorphic to a Lie subalgebra of a locally finite associative algebra.

(4) $L$ has a nontrivial module $V$ of finite type.

(5) $L$ has a non-trivial module $V$ such that both $V$ and its dual $V^{*}$ are integrable.

(6) [7] $L$ has a non-trivial inductive system of representations.

(7) [7] $U(L)$ has a proper ideal $Q$ such that $U(L) / Q$ is locally finite.

(8) [7] Every $x \in L$ is ad-algebraic, i.e. $P(\operatorname{ad} x)=0$ for some polynomial $P \neq 0$.

(9) $L$ has a non-zero ad-nilpotent element $x$.

(10) L has a non-zero Jordan element $x$ (i.e. $\left.(\operatorname{ad} x)^{3}=0\right)$.

(11) $L$ contains an sl $l_{2}$-triple $(x, h, y)$ such that $x$ is Jordan.

(12) L has a non-trivial finite $\mathbb{Z}$-grading.

(13) L has an abelian non-zero inner ideal.

(14) [16] L has a proper non-zero inner ideal.

(15) [2] L contains a finite dimensional simple grading subalgebra $S$ such that $L$ is $\Gamma$-graded where $\Gamma$ is a finite set of integral weights of $S$ containing the root system of $S$.

We will discuss the proof of this theorem in Section 3. Here we just make a few comments on some of the characterizations.

Property (5) was proved by Penkov and Serganova [35, Proposition 4.3] in the case of locally simple Lie algebras. We show that this holds in the general case by proving that (4) and (5) are equivalent.

Property (6) characterizes diagonal Lie algebras in terms of inductive systems of representations. This notion was introduced by Zalesski to study ideals in group algebras of locally finite groups (see for example, [36]) and proved to be useful in other areas as well. The lattices of inductive systems were studied for various direct limit groups and algebras, see for example [10]-[15], [30, 39, 40, 41]. Zalesski showed that inductive systems correspond to semiprimitive ideals in group algebras. A similar result holds for locally finite Lie algebras (see Theorem 2.16 below).

We previously conjectured that the property (7) can be strengthened as follows.

Conjecture 1.6. Let $L$ be as above. Then $L$ is diagonal if and only if

(16) $U(L)$ has a proper non-zero ideal.

In other words, $L$ is non-diagonal if and only if $U(L)$ is almost simple (i.e. $A(L)$ is a simple associative algebra).

In the case of a locally simple $L$, this conjecture has been recently proved by Penkov and Petukhov [34]. Note that for every finite dimensional Lie algebra $L$ the universal enveloping algebra $U(L)$ has a lot of ideals (for example, the annihilators of finite dimensional modules). Until recently, no-one was able to construct an example of a Lie algebra $L$ with almost simple $U(L)$. However, Conjecture 1.6 and its confirmation by Penkov and Petukhov in a special 
(and very important) case imply that $U(L)$ is almost simple for "almost all" simple locally finite Lie algebras $L$. This means that the following problem is justified.

Problem 1.7. Show that $U(L)$ is almost simple for all "generic" infinite dimensional simple Lie algebras $L$.

Properties (9)-(12) essentially appear in Hennig's paper [31] (as characterizations of simple Lie subalgebras of locally finite associative algebras).

Properties (13) and (14) give a characterization of diagonal Lie algebras in terms of inner ideals. Inner ideals were introduced by Benkart [21, 22] and proved to be useful in classifying simple Lie algebras, both of finite and infinite dimension, in zero and positive characteristic. They play a role similar to one-sided ideals of associative algebras in developing Artinian structure theory for Lie algebras $[27,28]$. They are also useful in constructing gradings of Lie algebras [29]. There is a complete classification of inner ideals in the case of simple finite dimensional [25] and finitary [26, 23] Lie algebras. Full classification of inner ideals of simple diagonal locally finite Lie algebras is not available but there are some partial results in [16]. In particular, inner ideals of diagonal locally semisimple algebras are classified in terms of systems of idempotents [16, Corollary 4.16]. Inner ideals can also be used to characterize the finitary simple Lie algebras. It follows from a general result, proved for nondegenerate Lie algebras by Draper, Fernndez Lpez, García and Gmez Lozano, that a simple locally finite Lie algebra has a proper minimal inner ideal if and only if it is finitary (see [24, Theorems 6.1 and 6.3]).

Property (15) relates diagonal Lie algebras to so-called root-graded Lie algebras. Rootgraded Lie algebras have been introduced by Berman and Moody for studying the toroidal algebras and Slodowy's intersection matrix algebras (see an important monograph [1] by Allison, Benkart, and Gao for references). It is shown in [2, Theorem 4.3] that each simple root-graded locally finite Lie algebra is diagonal and the converse is also true provided the notion of root-graded Lie algebras is generalized as follows. Let $\Delta$ be a root system of type $X_{n}(X=A, \ldots, G)$ and let $P(\Delta)$ be the group of integral weights of $\Delta$. Let $\Gamma$ be a subset of $P(\Delta)$ containing $\Delta$ and 0 . A Lie algebra $L$ is called $\Gamma$-graded if

(1) $L$ contains a finite-dimensional simple subalgebra $S=H \oplus \sum_{\mu \in \Delta} S_{\mu}$ whose root system is $\Delta$ relative to a Cartan subalgebra $H=S_{0}$,

(2) $L=\bigoplus_{\mu \in \Gamma} L_{\mu}$ where $L_{\mu}=\{x \in L \mid[h, x]=\mu(h) x$ for all $h \in H\}$,

(3) $L_{0}=\sum_{-\mu, \mu \in \Gamma \backslash\{0\}}\left[L_{-\mu}, L_{\mu}\right]$.

The subalgebra $S$ is called the grading subalgebra of $L$. Actually, it is proved in [2, Corollary 4.4] that any simple diagonal locally finite Lie algebra is $B C_{r}$-graded in the sense of [1] (which was also noticed in [3] in the case of diagonal locally finite-dimensional simple algebras of types $B, C, D)$. From this result and the results of Allison-Benkart-Gao [1] one can obtain another proof of the fact that any simple diagonal locally finite Lie algebra can be obtained as a Lie subalgebra of skew symmetric elements of a suitable associative algebra.

For analogues of Theorem 1.5 in positive characteristic we refer the reader to Hennig's recent paper [31]. The situation seems to be more difficult in that case and the best one can prove at the moment are the following two theorems (below $Z$ is the center of $[A, A]$ and $K=\operatorname{skew}(A)$ if $A$ has an involution).

Theorem 1.8. [31, Theorem 1] Let L be a simple locally finite Lie algebra over an algebraically closed field of characteristic $p>7$ or characteristic zero. Then the following are equivalent.

(1) $L$ is locally non-degenerate and there is a non-zero $x \in L$ with $(\operatorname{ad} x)^{p-1}=0$.

(2) $L=[A, A] / Z$ or $[K, K]$ for some simple locally finite associative algebra $A$. 
For the definition of locally nondegenerate, see Hennig's paper. We only note here that it is easy to check that simple locally finite Lie algebras over fields of characteristic zero are locally nondegenerate [31, Proposition 19].

Theorem 1.9. [31, Theorem 2] Let $L$ be a simple locally finite Lie algebra over an algebraically closed field of characteristic $p>3$. Suppose every element $x \in L$ is ad-algebraic (or equivalently, $L$ can be embedded into a locally finite associative algebra) and $p>2 d(L)-2$ where

$$
d(L)=\min \{\text { degree of the minimal polynomial of } \operatorname{ad}(x) \mid x \text { is not ad-nilpotent }\} .
$$

Then $L=[A, A] / Z$ or $[K, K]$ for some simple locally finite associative algebra $A$.

\section{LOCAL AND INDUCTIVE SYSTEMS}

Recall that a Lie algebra $L$ is called perfect if $[L, L]=L$. Similarly, an associative algebra $A$ is perfect if $A A=A$ (which is always true if $A$ contains an identity element). Let $L$ be a perfect finite-dimensional Lie algebra. Then its solvable radical $\operatorname{Rad} L$ annihilates every simple $L$-module and $L / \operatorname{Rad} L \cong Q_{1} \oplus \cdots \oplus Q_{n}$ is the sum of simple components $Q_{i}$. Denote by $V_{i}$ the first fundamental $Q_{i}$-module (so $V_{i}$ is natural and $Q_{i} \cong s l\left(V_{i}\right), s o\left(V_{i}\right), s p\left(V_{i}\right)$ if $Q_{i}$ is of classical type). The modules $V_{i}$ can be considered as $L$-modules in an obvious way and are called the natural $L$-modules. Assume that all $Q_{i}$ are of classical type. An $L$-module $V$ is called diagonal if each non-trivial composition factor of $V$ is a natural or co-natural module (i.e. dual to natural) of $L$. Otherwise $V$ is called non-diagonal. A diagonal $L$-module $V$ is called plain if all $Q_{i}$ are of type $A$ and each non-trivial composition factor of $V$ is a natural $L$-module. Let $L^{\prime}$ be another perfect finite dimensional Lie algebra containing $L$. If $W$ is an $L^{\prime}$-module we denote by $W \downarrow L$ the module $W$ restricted to $L$. Let $V_{1}^{\prime}, \ldots, V_{k}^{\prime}$ be the natural $L^{\prime}$-modules. The embedding $L \subseteq L^{\prime}$ is called diagonal (respectively plain) if $\left(V_{1}^{\prime} \oplus \cdots \oplus V_{k}^{\prime}\right) \downarrow L$ is a diagonal (respectively plain) $L$-module. By the minimal component rank of a perfect finite dimensional Lie algebra we mean the smallest rank of the simple components of $L / \operatorname{Rad} L$.

Note that we do not need to define diagonal embeddings for Lie algebras with non-classical components because they do not appear in the local systems we are going to use (see Remark 2.5 and Corollary 2.7).

Definition 2.1. A system of finite dimensional subalgebras $\mathfrak{L}=\left(L_{\alpha}\right)_{\alpha \in \Gamma}$ of a Lie (or associative) algebra $L$ is called a local system for $L$ if the following hold.

(1) $L=\bigcup_{\alpha \in \Gamma} L_{\alpha}$,

(2) for every pair $\alpha, \beta \in \Gamma$ there exists $\gamma \in \Gamma$ such that $L_{\alpha}, L_{\beta} \subseteq L_{\gamma}$.

Put $\alpha \leq \beta$ if $L_{\alpha} \subseteq L_{\beta}$. Then $\Gamma$ is a directed set and $L=\underline{\lim } L_{\alpha}$. We say that a local system is perfect (resp. semisimple) if it consists of perfect (resp. semisimple) subalgebras.

We list some elementary facts about simple locally finite Lie algebra $L$ and their local systems (see [4, Corollary 3.2, Theorem 3.2 and Lemma 3] or [6, Proposition 2.8].

Proposition 2.2. Let $L$ be a simple locally finite Lie algebra. Then the following holds.

(1) $L$ is not locally solvable.

(2) $L$ is locally perfect.

(3) If $\left(L_{\alpha}\right)_{\alpha \in \Gamma}$ is a local system for $L$ then for every $\alpha \in \Gamma$ there exists $\alpha^{\prime} \in \Gamma$ such that for all $\beta \geq \alpha^{\prime}$ one has $\operatorname{Rad} L_{\beta} \cap L_{\alpha}=0$.

Definition 2.3. A perfect local system $\left(L_{\alpha}\right)_{\alpha \in \Gamma}$ is called diagonal (resp. plain) if for all $\alpha \leq \beta$ the embedding $L_{\alpha} \subseteq L_{\beta}$ is diagonal (resp. plain). A simple locally finite Lie algebra $L$ 
is called diagonal (resp. plain) if it has a diagonal (resp. plain) local system. Otherwise, $L$ is called non-diagonal.

Note that plain locally finite Lie algebras are diagonal.

Definition 2.4. A perfect local system $\left(L_{\alpha}\right)_{\alpha \in \Gamma}$ is called conical if $\Gamma$ contains a minimal element 1 such that

(1) $L_{1} \subseteq L_{\alpha}$ for all $\alpha \in \Gamma$;

(2) $L_{1}$ is simple;

(3) for each $\alpha \in \Gamma$ the restriction of any natural $L_{\alpha}$-module to $L_{1}$ has a non-trivial composition factor.

By the rank of a conical system we mean the rank of the simple Lie algebra $L_{1}$.

Remark 2.5. Note that property (3) of the definition implies that for every $\alpha \in \Gamma$ and every simple component $S$ of a Levi subalgebra of $L_{\alpha}$ one has $\operatorname{rk} S \geq \operatorname{rk} L_{1}$. In particular, all these simple components are classical if $\mathrm{rk} L_{1} \geq 9$.

Proposition 2.6. [2, Proposition 3.1] Let L be a simple locally finite Lie algebra and let $\mathfrak{L}=\left(L_{\alpha}\right)_{\alpha \in \Gamma}$ be a perfect local system of $L$. Let $Q$ be a finite dimensional simple subalgebra of $L$. Fix any $\beta \in \Gamma$ such that $Q \subseteq L_{\beta}$. For $\gamma \geq \beta$, denote by $L_{\gamma}^{Q}$ the ideal of $L_{\gamma}$ generated by $Q$. Put $L_{1}^{Q}=Q$ and $\Gamma^{Q}=\{\gamma \in \Gamma \mid \gamma \geq \beta\} \cup\{1\}$. Then $\mathfrak{L}^{Q}=\left(L_{\alpha}^{Q}\right)_{\alpha \in \Gamma^{Q}}$ is a conical local system of $L$ and the following hold.

(1) Every natural $L_{\alpha}^{Q}$-module is the restriction of a natural $L_{\alpha}$-module. In particular, the embedding $L_{\alpha}^{Q} \subseteq L_{\alpha}$ is diagonal.

(2) If the local system $\mathfrak{L}$ is diagonal (resp. plain) then the local system $\mathfrak{L}^{Q}$ is diagonal (resp. plain).

Corollary 2.7. [2, Corollary 3.3] Simple locally finite Lie algebras have conical local systems of arbitrary large rank.

Remark 2.8. Similar results hold for locally finite associative algebras. In particular, every (involution) simple locally finite associative algebra $A$ has a conical (*-invariant) local system of subalgebras, see [2, Proposition 2.9]. Moreover, this system will be semisimple if $A$ is locally semisimple.

The following propositions describe some useful properties of conical local systems.

Proposition 2.9. [16, Corollary 2.11] Let L be a simple locally finite Lie algebra and let $\left(L_{\alpha}\right)_{\alpha \in \Gamma}$ be a conical local system for $L$. Then for every finite-dimensional simple subalgebra $Q$ of $L$ there exists $\alpha^{\prime} \in \Gamma$ such that for all $\beta \geq \alpha^{\prime}, Q \subseteq L_{\beta}$ and the restriction of every natural $L_{\beta}$-module $V$ to $Q$ has a non-trivial composition factor, i.e. $\left\{Q, L_{\beta} \mid \beta \geq \alpha^{\prime}\right\}$ is a conical local system of $L$.

Proposition 2.10. [16, Proposition 2.13] Let $L$ be a simple diagonal locally finite Lie algebra and let $\left(L_{\alpha}\right)_{\alpha \in \Gamma}$ be a conical local system of $L$. Then for every $n \in \mathbb{N}$ there is $\alpha^{\prime} \in \Gamma$ and a simple subalgebra $Q$ of $L$ with $\operatorname{rk} Q>n$ such that $Q \subseteq L_{\beta}$ for all $\beta \geq \alpha^{\prime}$ and $\left\{Q, L_{\beta} \mid \beta \geq \alpha^{\prime}\right\}$ is a conical diagonal local system of $L$ of rank $>n$.

Corollary 2.11. The algebra $s l_{\varphi}$ in Example 1.1(6) is diagonal if and only if there is an integer $k$ such that for all $n \geq k$ the $L_{n}$-module $V_{n+1}$ is diagonal (or equivalently, the embedding $L_{n} \rightarrow L_{n+1}$ is diagonal). In particular, the algebra sl $l_{a d}$ is non-diagonal.

Proof. This immediately follows from Proposition 2.10 . 
Theorem 2.12. [16, Theorem 2.14] Let $L$ be a simple diagonal locally finite Lie algebra and let $\left(L_{\alpha}\right)_{\alpha \in \Gamma}$ be a perfect local system for $L$. Assume that there is $\alpha \in \Gamma$, a non-zero $x \in L_{\alpha}$ and a natural number $k$ such that for all $\beta \geq \alpha$, the rank of $x$ is $\leq k$ on every natural $L_{\beta}$-module. Then $L$ is finitary.

Let $S$ be a finite dimensional Lie algebra. Denote by $\mathfrak{F}$ the set of all (two-sided) ideals in $U(S)$ of finite codimension. For any $X \in \mathfrak{F}$ the quotient $U(S) / X$ is a finite dimensional $S$-module under the left regular action, hence the notation $\operatorname{Irr}(U(S) / X)$ makes sense.

Theorem 2.13. [6, Theorem 3.4] Let $S$ be a perfect finite-dimensional Lie algebra, $\Phi$ a finite subset of $\operatorname{Irr} S$, and

$$
\mathfrak{F}(\Phi)=\{X \in \mathfrak{F} \mid \operatorname{Irr}(U(S) / X)=\Phi\} .
$$

Then $\mathfrak{F}(\Phi)$ is nonempty and has a smallest element $N(\Phi)$ and a largest element $M(\Phi)$, such that

$$
N(\Phi) \subseteq X \subseteq M(\Phi)
$$

for all $X \in \mathfrak{F}(\Phi)$. The algebra $U(S) / M(\Phi)$ is semisimple, while $M(\Phi) / N(\Phi)$ is nilpotent.

Proposition 2.14. Let $S$ be a perfect finite-dimensional Lie algebra and let $V$ be an $S$-module. Set $J=\operatorname{Ann}_{U(S)} V$ (annihilator of $V$ in $U(S)$ ). Then $V$ is of finite type if and only if $J$ has finite codimension in $U(S)$. Moreover, if $V$ is of finite type then $\operatorname{Irr}(V)=\operatorname{Irr}(U(S) / J)$.

Proof. If $U(S) / J$ is finite dimensional, then $\operatorname{dim} U(S) v<\infty$ for all $v \in V$, so $V$ is integrable. Moreover, the composition factors of $V$ correspond to the simple components of $U(S) / J$ so $V$ is of finite type.

Conversely, suppose that $V$ is of finite type. Put $\Phi=\operatorname{Irr}(V)$. Then $V$ is the union (more exactly, direct limit) of finite dimensional $S$-submodules $V_{\xi}$ with $\operatorname{Irr}\left(V_{\xi}\right)=\Phi$. Put $J_{\xi}=\operatorname{Ann}_{U(S)} V_{\xi}$. Then $\operatorname{Irr}\left(U(S) / J_{\xi}\right)=\operatorname{Irr}\left(V_{\xi}\right)=\Phi$. Therefore by Theorem $2.13, N(\Phi) \subseteq$ $J_{\xi} \subseteq M(\Phi)$ for all $\xi$. Since $J=\cap J_{\xi}$, we have $N(\Phi) \subseteq J \subseteq M(\Phi)$ so $J$ has finite codimension and $\operatorname{Irr}(U(S) / J)=\Phi$, as required.

Let $L$ be a locally perfect Lie algebra and let $\left(L_{\alpha}\right)_{\alpha \in \Gamma}$ be a perfect local system for $L$. Denote by $\mathfrak{I S}$ the set of inductive systems (of representations) of $L$ and by $\mathfrak{L F}$ the set of ideals of its universal enveloping algebra $U(L)$ with locally finite quotients.

Lemma 2.15. [6, Lemma 3.8] Let $L$ be as above and let $X \in \mathfrak{L F}$. Then the set

$$
\Phi(X)=\left\{\operatorname{Irr}\left(U\left(L_{\alpha}\right) / X \cap U\left(L_{\alpha}\right)\right)\right\}_{\alpha \in \Gamma}
$$

is an inductive system for $L$.

Define a map $f: \mathfrak{L F} \rightarrow \mathfrak{I S}$, by setting $f(X)=\Phi(X)$ where $X \in \mathfrak{L} \mathfrak{F}$. Denote by $\mathfrak{L} \mathfrak{F}(\Phi)$ the inverse image of the inductive system $\Phi$.

Theorem 2.16. [6, Theorem 3.9] Let $L$ be a locally perfect Lie algebra and let $f: \mathfrak{L F} \rightarrow \mathfrak{I S}$ be the above map. Then for every inductive system $\Phi$ the set $\mathfrak{L F}(\Phi)$ is nonempty and has a smallest element $N(\Phi)$ and a largest element $M(\Phi)$, such that

$$
N(\Phi) \subseteq X \subseteq M(\Phi)
$$

for all $X \in \mathfrak{L F}(\Phi)$. The algebra $U(L) / M(\Phi)$ is semiprimitive and the algebra $M(\Phi) / N(\Phi)$ is locally nilpotent. Moreover, the map $f$ produces a bijection between semiprimitive ideals in $\mathfrak{L} \mathfrak{F}$ and inductive systems for $L$ (the inverse map is given by $\Phi \mapsto M(\Phi)$ ). 
Proposition 2.17. Let $L$ be a locally perfect Lie algebra and let $\left(L_{\alpha}\right)_{\alpha \in \Gamma}$ be a perfect local system for $L$. Let $V$ be an $L$-module and let $J=\operatorname{Ann}_{U(L)} V$. Then $V$ is of finite type if and only if the algebra $U(L) / J$ is locally finite. Moreover, if $V$ is of finite type then

$$
\operatorname{Irr}\left(V \downarrow L_{\alpha}\right)=\operatorname{Irr}\left(U\left(L_{\alpha}\right) / J \cap U\left(L_{\alpha}\right)\right)
$$

for all $\alpha$ and $\Phi(V)=\left(\operatorname{Irr}\left(V \downarrow L_{\alpha}\right)\right)_{\alpha \in \Gamma}=\Phi(J)$ is an inductive system for $L$.

Proof. Put $J_{\alpha}=\operatorname{Ann}_{U\left(L_{\alpha}\right)} V=J \cap U\left(L_{\alpha}\right)$. Obviously, $U(L) / J$ is locally finite if and only if $U\left(L_{\alpha}\right) / J_{\alpha}$ is finite dimensional for all $\alpha$. Now the result follows from Proposition 2.14 and Lemma 2.15 .

\section{Proof of the Main TheOREM}

In this section we prove Theorem 1.5. Throughout this section, $L$ is a simple locally finite Lie algebra and $\left(L_{\alpha}\right)_{\alpha \in \Gamma}$ is a conical local system for $L$ of rank $\geq 9$ (see Corollary 2.7).

Claim 3.1. (1) $\Leftrightarrow(2) \Leftrightarrow(3)$

Proof. We refer the reader to [2] for details.

Claim 3.2. (3) $\Leftrightarrow(4) \Leftrightarrow(6) \Leftrightarrow(7)$

Proof. (3) $\Rightarrow(4)$ Suppose that $L$ is isomorphic to a Lie subalgebra of a locally finite associative algebra $A$. Let $B$ be the associative subalgebra of $A$ generated by $L$. Consider $B$ as an $L$ module $V$ with respect to the left regular action. Then $V$ is a non-trivial $L$-module of finite type.

$(4) \Rightarrow(6)$ Suppose $V$ is a non-trivial $L$-module of finite type. Then by Proposition 2.17, $\Phi(V)$ is a non-trivial inductive system of representations for $L$.

$(6) \Rightarrow(7)$ This follows from Theorem 2.16.

$(7) \Rightarrow(3)$ Follows from the fact that $L$ is obviously a Lie subalgebra of $U(L) / Q$.

Claim 3.3. (4) $\Leftrightarrow(5)$

Proof. Let $V$ be a non-trivial $L$-module of finite type. Then $V$ is integrable by definition and we need to show that $V^{*}$ is integrable. Let $S$ be a finite dimensional subalgebra of $L$. We need to show that $V^{*}$ is integrable as $S$-module. By taking a larger subalgebra if necessary one can assume that $S$ is perfect (see Proposition 2.2). Let $J=\operatorname{Ann}_{U(S)} V$. Then by Proposition 2.14, $J$ has finite codimension. Let $\eta: U(S) \rightarrow U(S)$ be the antipode of $U(S)$ (i.e. the antiautomorphism induced by the map $x \mapsto-x$ for all $x \in S$ ). Then $\eta(J)$ obviously annihilates $V^{*}$, so $\operatorname{Ann}_{U(S)} V^{*}$ is of finite codimension. Therefore by Proposition $2.14, V^{*}$ is of finite type as $S$-module.

Conversely, suppose that both $V$ and $V^{*}$ are integrable but $V$ is of infinite type. Fix any $\alpha$ such that $\operatorname{Irr}\left(V \downarrow L_{\alpha}\right)$ is infinite. Let $S$ be any Levi subalgebra of $L_{\alpha}$. Since $\operatorname{Rad} L_{\alpha}$ annihilates all irreducible $L_{\alpha}$-modules, the set $\operatorname{Irr}(V \downarrow S)$ is infinite. Note that $V$ is the union of finite dimensional semisimple $S$-submodules. Fix any Cartan subalgebra $H$ of $S$ and the root system. Let $\rho$ be the maximal root of $S$ and let $h$ be the corresponding element in $H$. Then $h$ has an infinite number of distinct eigenvalues $\lambda_{1}, \lambda_{2}, \ldots$ on $V$. Let $v_{1}, v_{2}, \ldots$ be corresponding eigenvectors. Since all $\lambda_{i}$ are distinct, the $v_{i}$ are linearly independent. Consider a function $f \in V^{*}$ such that $f\left(v_{i}\right)=1$ for all $i$. We claim that $U(\mathbb{F} h) f$ is not finite dimensional. Indeed, suppose that $U(\mathbb{F} h) f$ is finite dimensional. Then there is a polynomial $p \neq 0$ such that $p(h) f=0$. Therefore for all $i=1,2, \ldots$,

$$
0=(p(h) f)\left(v_{i}\right)=f\left(p^{\eta}(h) v_{i}\right)=f\left(p^{\eta}\left(\lambda_{i}\right) v_{i}\right)=p^{\eta}\left(\lambda_{i}\right),
$$


which is a contradiction. Therefore $U(\mathbb{F} h) f$ is not finite dimensional and hence $V^{*}$ is not integrable, which contradicts the assumption.

Claim 3.4. (3) $\Leftrightarrow(8)$

Proof. We repeat our argument from [6]. Suppose that (3) holds, i.e. $L$ is a Lie subalgebra of a locally finite associative algebra $A$. Then for each $x \in L$ there exists a polynomial $g_{x}$ such that $g_{x}(x)=0$ in $A$. Hence $g_{x}\left(l_{x}\right)=g_{x}\left(r_{x}\right)=0$ in $\operatorname{End}_{\mathbb{F}} A$ where $l_{x}: a \mapsto x a, r_{x}: a \mapsto a x$ are endomorphisms of $A$. Since $l_{x} r_{x}=r_{x} l_{x}$ and ad $x=l_{x}-r_{x}$, there exists a polynomial $f_{x}$ such that $f_{x}(\operatorname{ad} x)=0$ in $\operatorname{End}_{\mathbb{F}} A$. Hence $f_{x}(\operatorname{ad} x)=0$ in $\operatorname{End}_{\mathbb{F}} L$ and (8) holds.

Suppose now that each ad $x$ is algebraic. Denote by $\operatorname{Ad} L$ the associative subalgebra of $\operatorname{End}_{\mathbb{F}} L$ generated by all ad $x, x \in L$. Since $L$ is simple, its center is trivial, so the map $x \mapsto \operatorname{ad} x$ is an embedding of $L$ into $\operatorname{Ad} L$. We wish to show that $\operatorname{Ad} L$ is locally finite. Let $B$ be a finitely generated subalgebra of $\operatorname{Ad} L$. We have to prove that $B$ is finite-dimensional. Without loss of generality one can assume that $B$ is generated by ad $x_{1}, \ldots$, ad $x_{m}$ where $x_{1}, \ldots, x_{m}$ are linearly independent elements of $L$. Let $x_{1}, \ldots, x_{m}, \ldots, x_{n}$ be a basis of the Lie subalgebra of $L$ generated by $x_{1}, \ldots, x_{m}$, and let $C$ be the subalgebra of Ad $L$ generated by ad $x_{1}, \ldots$, ad $x_{n}$. It is clear that $B \subseteq C$. Since

$$
\left[\operatorname{ad} x_{i}, \operatorname{ad} x_{j}\right]=\operatorname{ad}\left[x_{i}, x_{j}\right] \in\left\langle\operatorname{ad} x_{1}, \ldots, \operatorname{ad} x_{n}\right\rangle_{F},
$$

$C$ is a linear subspace of the linear space $V$ generated by the elements of type

$$
\left(\operatorname{ad} x_{1}\right)^{k_{1}} \ldots\left(\operatorname{ad} x_{n}\right)^{k_{n}} \text {. }
$$

Since for each $i$ there exists a polynomial $f_{i}$ such that $f_{i}\left(\operatorname{ad} x_{i}\right)=0$, the space $V$ is finitedimensional. Hence $B$ is finite-dimensional, as required.

We will need the following lemma.

Lemma 3.5. Let $L$ be a simple locally finite Lie algebra. Suppose that there is a non-zero $x \in L$ such that $(\operatorname{ad} x)^{n}=0$. Then there is a finite dimensional semisimple subalgebra $S$ of $L$ and a non-zero element $s \in S$ such that $(\operatorname{ad} s)^{n}=0$ on L. Moreover, there exist $y, h \in S$ such that $(s, h, y)$ is an $s l_{2}$-triple of $L$.

Proof. Let $\left(L_{\alpha}\right)_{\alpha \in \Gamma}$ be a conical local system for $L$ of rank $\geq 9$. Fix any $\alpha$ such that $x \in L_{\alpha}$. By taking a larger subalgebra if necessary, by Proposition 2.2(3), one can assume that $x \notin \operatorname{Rad} L_{\alpha}$. Let $S$ be any Levi subalgebra of $L_{\alpha}$. Then $x=s+r$ for some non-zero $s \in S$ and some $r \in \operatorname{Rad} L_{\alpha}$. Let $\beta \geq \alpha$. Consider $L_{\beta}$ as an $L_{\alpha}$-module with respect to the adjoint action. Let $W$ be any composition factor of this module. Since $L_{\alpha}$ is perfect, $r$ acts as 0 on $W$, so ad $x=\operatorname{ad} s$ on $W$. Therefore $(\operatorname{ad} s)^{n}=0$ on $W$. Since $S$ is semisimple, $(\operatorname{ad} s)^{n}=0$ on $L_{\beta}$ for all $\beta \geq \alpha$. This implies that $(\operatorname{ad} s)^{n}=0$ on $L$. Since $s$ is a nilpotent element of $S$, the "moreover" part follows from the Jacobson-Morozov Theorem.

Remark 3.6. It follows from the proof of Lemma 3.5 that every simple locally finite Lie algebra $L$ over $\mathbb{F}$ is non-degenerate, i.e. it has no non-zero elements $x$ with $(\operatorname{ad} x)^{2}=0$. Indeed, otherwise there is a semisimple subalgebra $S$ of $L$ and a non-zero $s \in S$ with $(\operatorname{ad} s)^{2}=0$, which contradicts to Jacobson-Morozov Theorem.

Claim 3.7. (9) $\Leftrightarrow(10) \Leftrightarrow(11) \Leftrightarrow(12) \Leftrightarrow(13)$

Proof. (9) $\Rightarrow(10)$ This follows from the Kostrikin's Descent Lemma [33, Lemma 1.1, p.31] (if $x$ is an ad-nilpotent element of a Lie algebra $L$ of index $n \geq 4$, then for every $a \in L$, $(\operatorname{ad} x)^{n-1}(a)$ is an ad-nilpotent element of index at most $\left.n-1\right)$.

$(10) \Rightarrow(11)$ This follows from Lemma 3.5. 
$(11) \Rightarrow(12)$ Let $(x, h, y) \subset L$ be an $s_{2}$-triple with $(\operatorname{ad} x)^{3}=0$ and let $S=\operatorname{span}\{x, h, y\}$. Consider $L$ as an $S$-module with respect to the adjoint action. Since $S \cong s l_{2}$ is semisimple, $L$ is the (infinite) direct sum of some simple modules $W_{\xi}$. As $x^{3}=0$ on the $S$-module $V$, the only possibilities for $W_{\xi}$ are to be either trivial or natural or adjoint $S$-module, so the only possibilities for the weights of $h$ on $W_{\xi}$ are $-2,-1,0,1,2$. As each $W_{\xi}$ is the direct some of its weight spaces with respect to $h$ we get the following non-trivial finite $\mathbb{Z}$-grading on $L$ :

$$
L=L_{-2} \oplus L_{-1} \oplus L_{0} \oplus L_{1} \oplus L_{2}
$$

where $L_{i}=\{a \in L \mid[h, a]=i a\}$.

$(12) \Rightarrow(13)$ Suppose $L$ has a non-trivial finite $\mathbb{Z}$-grading

$$
L=L_{-n} \oplus \cdots \oplus L_{-1} \oplus L_{0} \oplus L_{1} \oplus \cdots \oplus L_{n}
$$

Without loss of generality one can assume that $L_{n} \neq 0$. Put $B=L_{n}$. Then

$$
[B,[B, L]]=\left[L_{n},\left[L_{n}, L\right]\right] \subseteq L_{n}=B
$$

so $B$ is a non-zero inner ideal of $L$. Moreover $[B, B]=\left[L_{n}, L_{n}\right]=0$, so $B$ is abelian.

$(13) \Rightarrow(9)$ Suppose $L$ has an abelian non-zero inner ideal $B$. Then for every $x \in B$ one has $[x,[x,[x, L]]] \subseteq[x, B]=0$, so $(\operatorname{ad} x)^{3}=0$ and $x$ is ad-nilpotent.

Claim 3.8. (1) $\Leftrightarrow(14)$

Proof. This is obvious in one direction but much more difficult in the other. We refer the reader to [16] for details.

Claim 3.9. (1) $\Leftrightarrow(15)$

Proof. We refer the reader to [2, Section 4] for details.

It remains to link the group $(1) \Leftrightarrow \cdots \Leftrightarrow$ (8) with the group $(9) \Leftrightarrow \cdots \Leftrightarrow(13)$.

Claim 3.10. (8) $\Rightarrow(9)$

Proof. Suppose that every element of $L$ is ad-algebraic. Let $S$ be any finite dimensional simple subalgebra of $L$ (which exists because of Corollary 2.7) and let $x$ be any non-zero nilpotent element of $S$. Then $\operatorname{ad} x$ is locally nilpotent on $L$. Since ad $x$ is algebraic, it must be nilpotent.

There are several ways to go in the other direction. One of them is to use Zelmanov's classification of simple Lie algebras with finite gradings [38] and to prove that (12) implies (2) (see Hennig's paper [31] for details). Another way is to use representation-theoretic technique, which works as follows.

Claim 3.11. (11) $\Rightarrow(4)$

Proof. Suppose $L$ contains an $s l_{2}$-triple $(x, h, y)$ such that $x$ is Jordan. Denote by $V$ the adjoint $L$-module $L$. As above, $V$ is the direct sum of the $h$-eigenspaces with eigenvalues $-2,-1,0,1,2$. The module $V$ is obviously integrable as the union of finite dimensional submodules $L_{\alpha}$. We wish to show that $V$ is of finite type (so (4) holds). Assume this not the case. Then there is $\alpha \in \Gamma$ and $v \in V$ such that $\operatorname{Irr}\left(V \downarrow L_{\alpha}\right)$ is infinite. Without loss of generality one can assume that $h \in L_{\alpha}$. As $h$ is a semisimple element, there is a Levi subalgebra $S$ of $L_{\alpha}$ such that $h \in S$. Note that $\Phi=\operatorname{Irr}(V \downarrow S)=\operatorname{Irr}\left(V \downarrow L_{\alpha}\right)$ is infinite, so there is an infinite number of distinct integral dominant weights $\lambda_{1}, \lambda_{2}, \ldots$ such that $W\left(\lambda_{i}\right) \in \Phi$ where $W\left(\lambda_{i}\right)$ is the simple module of highest weight $\lambda_{i}$. It is not difficult to see that one can find a sufficiently large $i$ such that the module $W\left(\lambda_{i}\right)$ contains a weight $\mu$ with $|\mu(h)|>2$, which is a contradiction. 


\section{REFERENCES}

[1] B. Allison, G. Benkart, Y. Gao, Lie algebras graded by the root systems $B C_{r}, r \geq 2$. Mem. Amer. Math. Soc. 158 (2002), no. $751, \mathrm{x}+158$ pp.

[2] Y.A. Bahturin, A.A. Baranov, A.E. Zalesski, Simple Lie subalgebras of locally finite associative algebras, J. Algebra 281 (2004), 225-246.

[3] Y. Bahturin, G. Benkart, Some constructions in the theory of locally finite simple Lie algebras, J. Lie Theory 14 (2004) 243-270.

[4] Y.A. Bahturin, H. Strade, Locally finite-dimensional simple Lie algebras, Russian Acad. Sci. Sb. Math. 81 (1995), no. 1, 137-161.

[5] Y. Bahturin, H. Strade, Some examples of locally finite simple Lie algebras, Arch. Math., 65 (1995) (3-4), 209-213.

[6] A.A. Baranov, Diagonal locally finite Lie algebras and a version of Ado's Theorem, J. Algebra 199 (1998), $1-39$.

[7] A.A. Baranov, Simple diagonal locally finite Lie algebras, Proc. London Math. Soc., 77 (1998), 362-386.

[8] A.A. Baranov, Finitary simple Lie algebras. J. Algebra 219 (1999), 299-329.

[9] A.A. Baranov, Classification of the direct limits of involution simple associative algebras and the corresponding dimension groups, J. Algebra 381 (2013), 73-95.

[10] A.A. Baranov, A.S. Kleshchev, Maximal ideals in modular group algebras of the finitary symmetric and alternating groups, Trans. Amer. Math. Soc. 351 (1999), 595-617.

[11] A.A. Baranov, A.S. Kleshchev and A.E. Zalesskii, Asymptotic results on modular representations of symmetric groups and almost simple modular group algebras, J. Algebra 219 (1999), 506-530.

[12] A.A. Baranov, A.A. Osinovskaya, I.D. Suprunenko, Modular representations of the special linear groups with small weight multiplicities, J. Algebra 397 (2014), 225 - 251.

[13] A.A. Baranov and I.D. Suprunenko, Branching rules for modular fundamental representations of symplectic groups, Bull. London Math. Soc. 32 (2000), 409-420.

[14] A.A. Baranov and I.D. Suprunenko, Minimal inductive systems of modular representations for naturally embedded algebraic and finite groups of type A, Comm. Algebra 29 (2001), 3117-3133.

[15] A.A. Baranov and I.D. Suprunenko, Modular branching rules for 2-column diagram representations of general linear groups, Journal of Algebra and its Applications Vol. 4 , No. 5 (2005), 489-515.

[16] A.A. Baranov, J. Rowley, Inner ideals of simple locally finite Lie algebras, J. Algebra 379 (2013), 11-30.

[17] A.A. Baranov, H. Strade, Finitary Lie algebras, J. Algebra 254 (2002), 173-211.

[18] A.A. Baranov, A.E Zalesski, Plain representations of Lie algebras, J. London Math Soc. 63 (2001), $571-591$.

[19] A.A. Baranov, A.E. Zalesski, Quasiclassical Lie algebras, J. Algebra 243 (2001), 264-293.

[20] A.A. Baranov, A.G. Zhilinskii, Diagonal direct limits of simple Lie algebras, Comm. in Algebra 27 (1999), 2749-2766.

[21] G. Benkart, The Lie inner ideal structure of associative rings, J. Algebra 43 (1976), 561-584.

[22] G. Benkart, On inner ideals and ad-nilpotent elements of Lie algebras, Trans. Amer. Math. Soc. 232 (1977), 61-81.

[23] G. Benkart, A. Fernndez Lpez, The inner ideal structure of associative rings revisited, Comm. Algebra, 37 (2009), 3833-3850.

[24] C. Draper, A. Fernndez Lpez, E. García, and M. Gmez Lozano, The socle of a nondegenerate Lie algebra, J.Algebra 319 (2008), 2372-2394.

[25] C. Draper, A. Fernndez Lpez, E. Garca, and M. Gmez Lozano, The inner ideals of the simple finite dimensional Lie algebras, J. Lie Theory 22 (2012), no. 4, 907-929.

[26] A. Fernndez Lpez, E. García, M. Gmez Lozano, Inner ideals of finitary simple Lie algebras, J. Lie Theory 16 (2006), 97-114.

[27] A. Fernndez Lpez, E. García, M. Gmez Lozano, An Artinian theory for Lie algebras. J. Algebra 319 (2008), no. 3, 938-951.

[28] A. Fernndez Lpez, E. García, M. Gmez Lozano, Inner ideal structure of nearly Artinian Lie Algebras, Proc. Amer. Math Soc. 137 (2009), 1-9.

[29] A. Fernndez Lpez, E. García, M. Gmez Lozano, E. Neher, A construction of gradings of Lie algebras, Int. Math. Res. Not. IMRN 2007, no. 16, Art. ID rnm051, 34 pp.

[30] B. Hartley and A. Zalesskii, Confined subgroups of locally finite simple groups and ideals of their group rings, J. London Math. Soc. (2) 55(1997), 210 - 230.

[31] J. Hennig, Simple locally finite dimensional Lie algebras in positive characteristic, J. Algebra 413 (2014), 270-288. 
[32] I.N. Herstein, Lie and Jordan structures in simple associative rings, Bull. Amer. Math. Soc. 67 (1961) $517-531$

[33] A.I. Kostrikin. Around Burnside (transl. J. Wiegold), Ergebnisse der Mathematik und ihrer Grenzgebiete, 20 Berlin, Springer-Verlag (1990).

[34] I. Penkov, A. Petukhov, On ideals in the enveloping algebra of a locally simple Lie algebra, Int. Math. Res. Notices (2014), doi: 10.1093/imrn/rnu085

[35] I. Penkov, V. Serganova, Categories of integrable $s l(\infty)-, o(\infty)-, s p(\infty)$-modules, In: Representation theory and mathematical physics, Contemp. Math., 557, Amer. Math. Soc., Providence, RI, 2011, pp. $335-357$.

[36] A.E. Zalesskii, Group rings of simple locally finite groups, In: Finite and locally finite groups, Kluwer, Dordrecht, 1995, pp. 219-246.

[37] A. Zalesskii, Direct limits of finite dimensional algebras and finite groups, In: Trends in Ring Theory: Proceedings of a Conference at Miskolc, Hungary, July 15-20, 1996, American Mathematical Society for the Canadian Mathematical Society, Providence, RI, 1998.

[38] E.I. Zelmanov, Lie algebras with a finite grading, Mat. Sb. (N.S.) 124(166) (3) (1984) 353-392.

[39] A.G. Zhilinskii, Coherent systems of representations of inductive families of simple complex Lie algebras, (Russian) preprint of Academy of Belarussian SSR, ser. 38(438), Minsk, 1990.

[40] A.G. Zhilinskii, Coherent finite-type systems of inductive families of non-diagonal inclusions, (Russian) Dokl. Acad. Nauk Belarusi 36:1(1992), 9-13.

[41] A.G. Zhilinskii, On the lattice of ideals in the universal enveloping algebra of a diagonal Lie algebra, preprint, Minsk, 2011.

Department of Mathematics, University of Leicester, Leicester, LE1 7RH, UK

E-mail address: ab155@le.ac.uk 